\title{
Muséologies
}

Les cahiers d'études supérieures

\section{Jean-Louis Cohen}

\section{Alessandra Mariani}

Volume 5, numéro 2, printemps 2011

URI : https://id.erudit.org/iderudit/1033516ar

DOI : https://doi.org/10.7202/1033516ar

Aller au sommaire du numéro

Éditeur(s)

Association Québécoise de Promotion des Recherches Étudiantes en Muséologie (AQPREM)

ISSN

1718-5181 (imprimé)

1929-7815 (numérique)

Découvrir la revue

Citer ce document

Mariani, A. (2011). Jean-Louis Cohen. Muséologies, 5(2), 78-101.

https://doi.org/10.7202/1033516ar

Ce document est protégé par la loi sur le droit d'auteur. L’utilisation des services d'Érudit (y compris la reproduction) est assujettie à sa politique d'utilisation que vous pouvez consulter en ligne.

https://apropos.erudit.org/fr/usagers/politique-dutilisation/
Cet article est diffusé et préservé par Érudit.

Érudit est un consortium interuniversitaire sans but lucratif composé de l’Université de Montréal, l'Université Laval et l'Université du Québec à Montréal. Il a pour mission la promotion et la valorisation de la recherche. https://www.erudit.org/fr/ 


\title{
Jean-Louis Cohen
}

\author{
Entretien avec Alessandra Mariani
}

Né à Paris en 1949, Jean-Louis Cohen est architecte et historien. Ses recherches ont porté notamment sur l'architecture et l'urbanisme du XXe siècle en France, en Allemagne, en Italie, en Russie et aux Etats-Unis, les formes de l'internationalisation et les cultures régionales, la modernisation de la forme urbaine à Paris et l'urbanisme dans le Maroc du protectorat français. Entre 1998 et 2003, Jean-Louis Cohen a élaboré et conduit le projet de Cité de l'architecture et du patrimoine dans le palais de Chaillot de Paris, dirigeant l'Institut français d'architecture et le musée des Monuments français.

Depuis 1994, il occupe la chaire Sheldon H. Solow en histoire de l'architecture à l'Institute of Fine Arts de l'Université de New York. Il a conçu de multiples expositions au centre Pompidou, au pavillon de I'Arsenal, au Centre Canadien d'Architecture, à l'Institut français d'architecture et au MoMA.

Il a publié de nombreux ouvrages, parmi lesquels Le Corbusier et la mystique de I'URSS (BruxellesLiège, Mardaga, 1987); Des fortifs au périf, Paris: les seuils de la ville (avec André Lortie, Paris, Picard, 1992); Scènes de la vie future, l'architecture européenne et la tentation de l'Amérique 1893-1960 (Paris, Flammarion, 1995); Casablanca, mythes et figures d'une aventure urbaine (avec Monique Eleb, Paris, Hazan, 1998); Le Corbusier, la planète comme chantier (Paris, Éditions Textuel, 2005); Mies van der Rohe (Paris, Hazan, 2007); New York (Paris, CitadellesMazenod, 2008). 


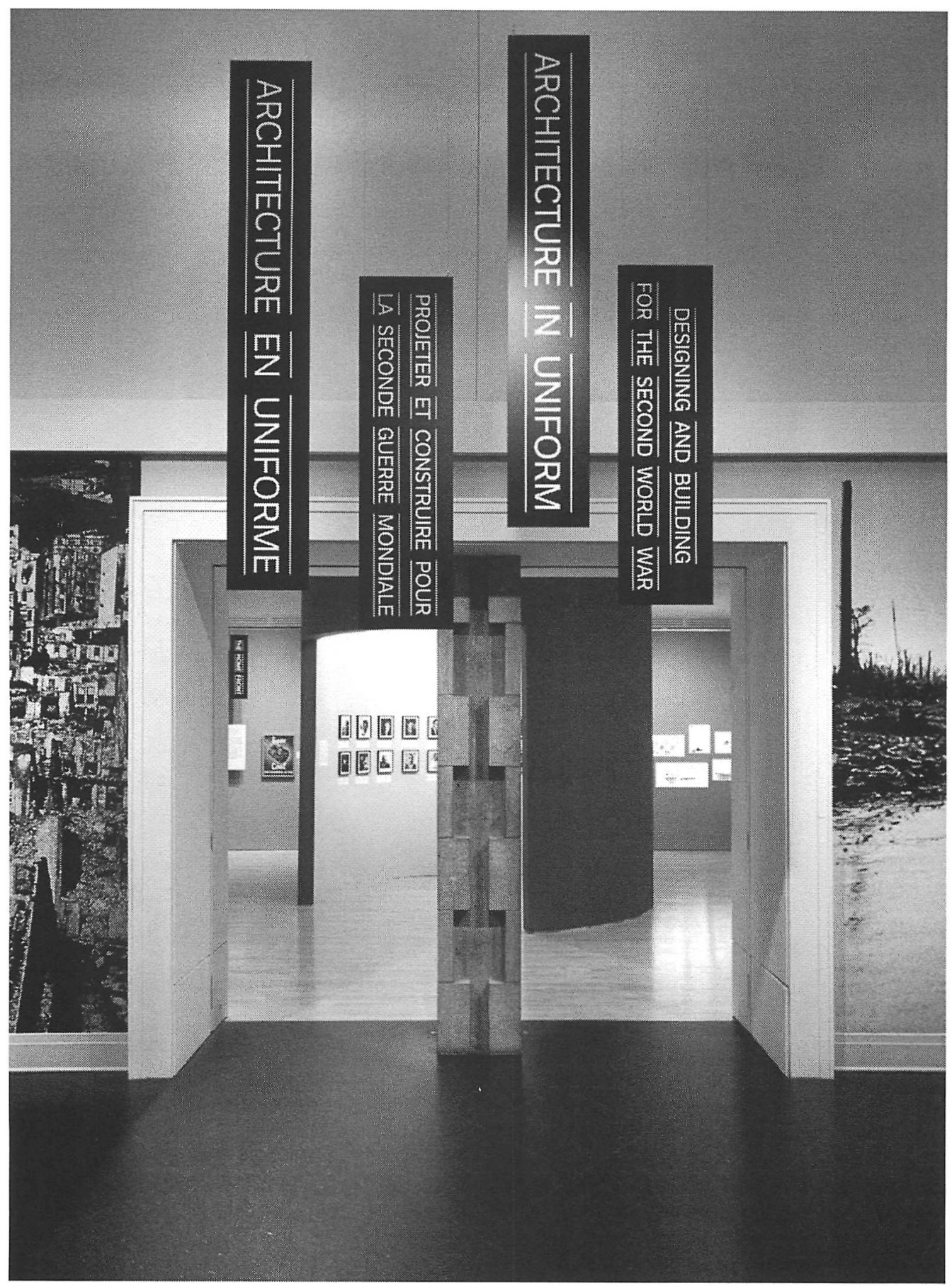

Vue de l'exposition du CCA, Architecture en uniforme: projeter et construire pour la Seconde Cuerre mondiale. Centre Canadien d'Architecture, Montréal. 


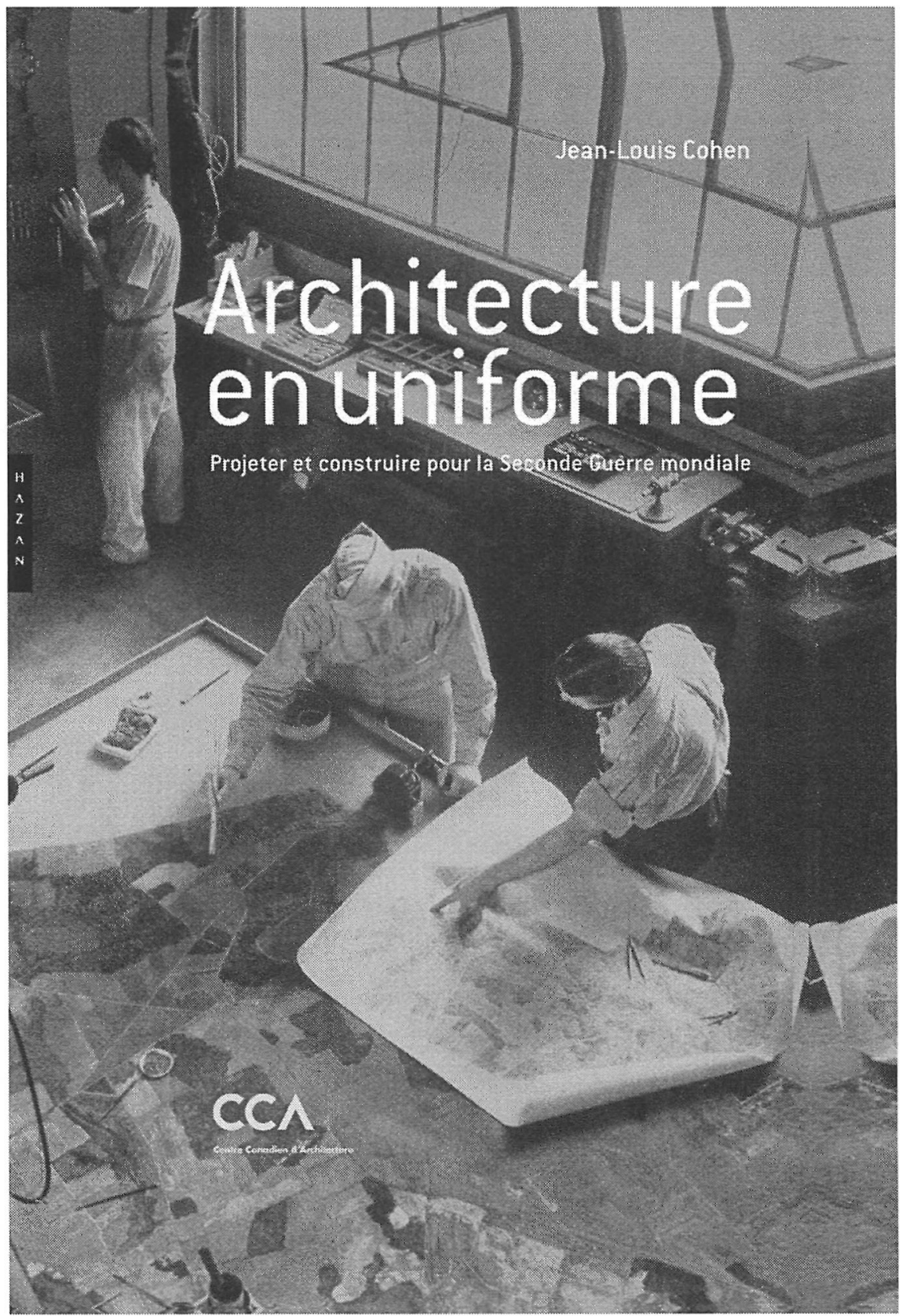

Ouvrage Architecture en uniforme:

projeter et construire pour la Seconde Guerre

mondiale (2011). o CCA / Hazan 
Le Centre Canadien d'Architecture présente Architecture en uniforme: Projeter et construire pour la Seconde Guerre mondiale du 13 avril au 18 septembre 2011. L'exposition, une mise en forme des recherches de Jean-Louis Cohen, vient combler un silence de l'histoire de l'architecture qui avait célébré jusqu'ici l'essor idéologique et fonctionnaliste du Mouvement moderne en occultant une période importante de son apogée : celle de l'activité et de la production des architectes au cours de la Seconde Guerre.

Ce nouveau récit monté autour de huit thèmes - Le front domestique, Guerre aux villes, Produire la production de guerre, Architectures mobiles, La forteresse Europe, Camoufler ou le dessin de l'invisible, Quatre macro-projets et De la guerre à la paix - fait état des innovations matérielles, technologiques et systémiques mises en place en temps de guerre tout en révélant celles qui ont eu - et qui ont toujours - un impact sur l'architecture et l'urbanisme contemporains. L'exposition qui s'ingénie à reproduire autant le dynamisme des idées que leur réalisation, s'appuie sur une grande quantité de documents graphiques et photographiques du Centre Canadien d'Architecture et d'institutions internationales comme d'extraits filmiques de l'Office national du film du Canada dans cette période. 
Arsenal de chars de Chrysler, Warren Township, Michigan, par Albert Kahn Associates, 1941. Photographie de Hedrich-Blessing. (c) Chicago History Museum, HB-06539-C

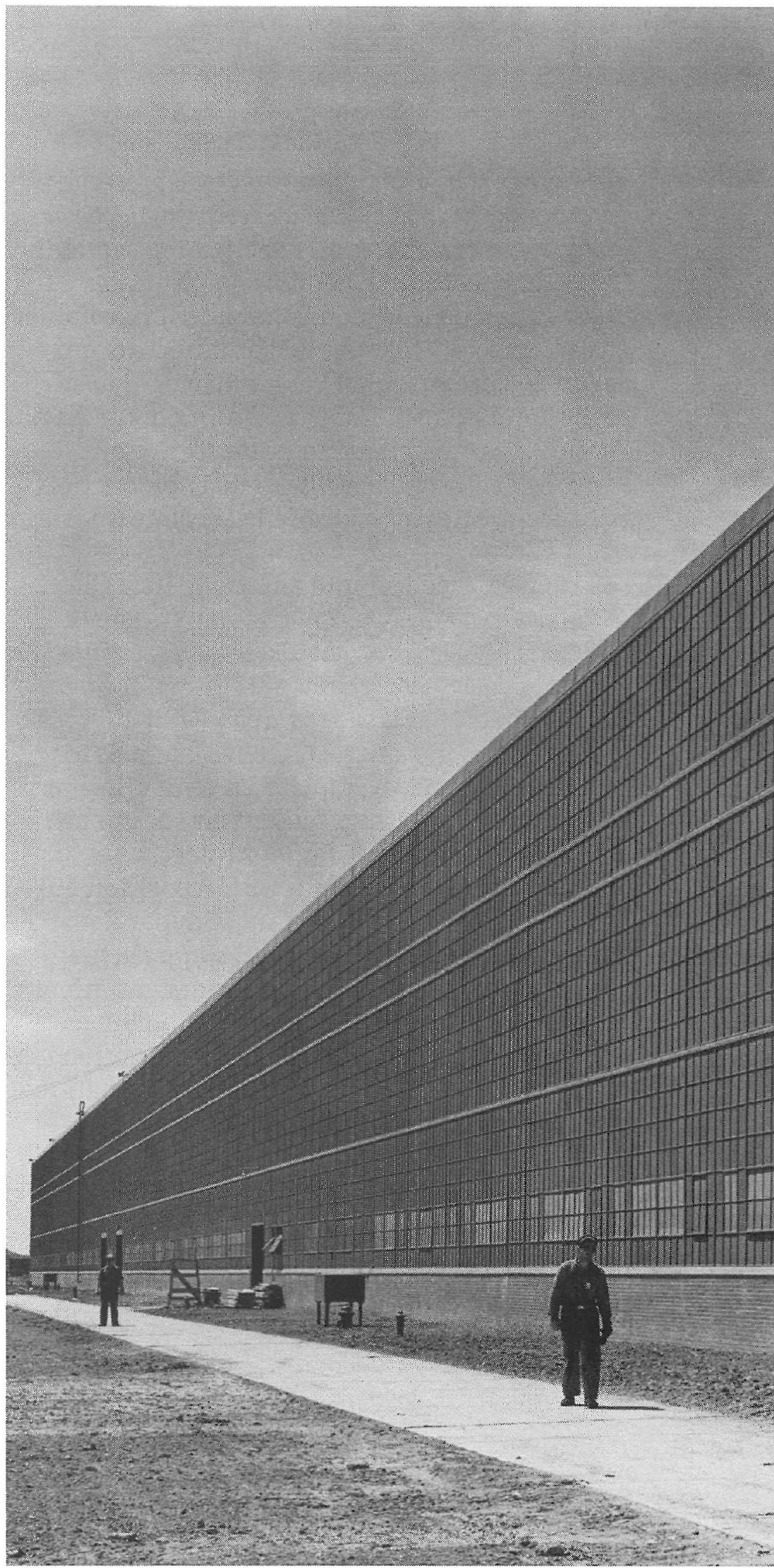




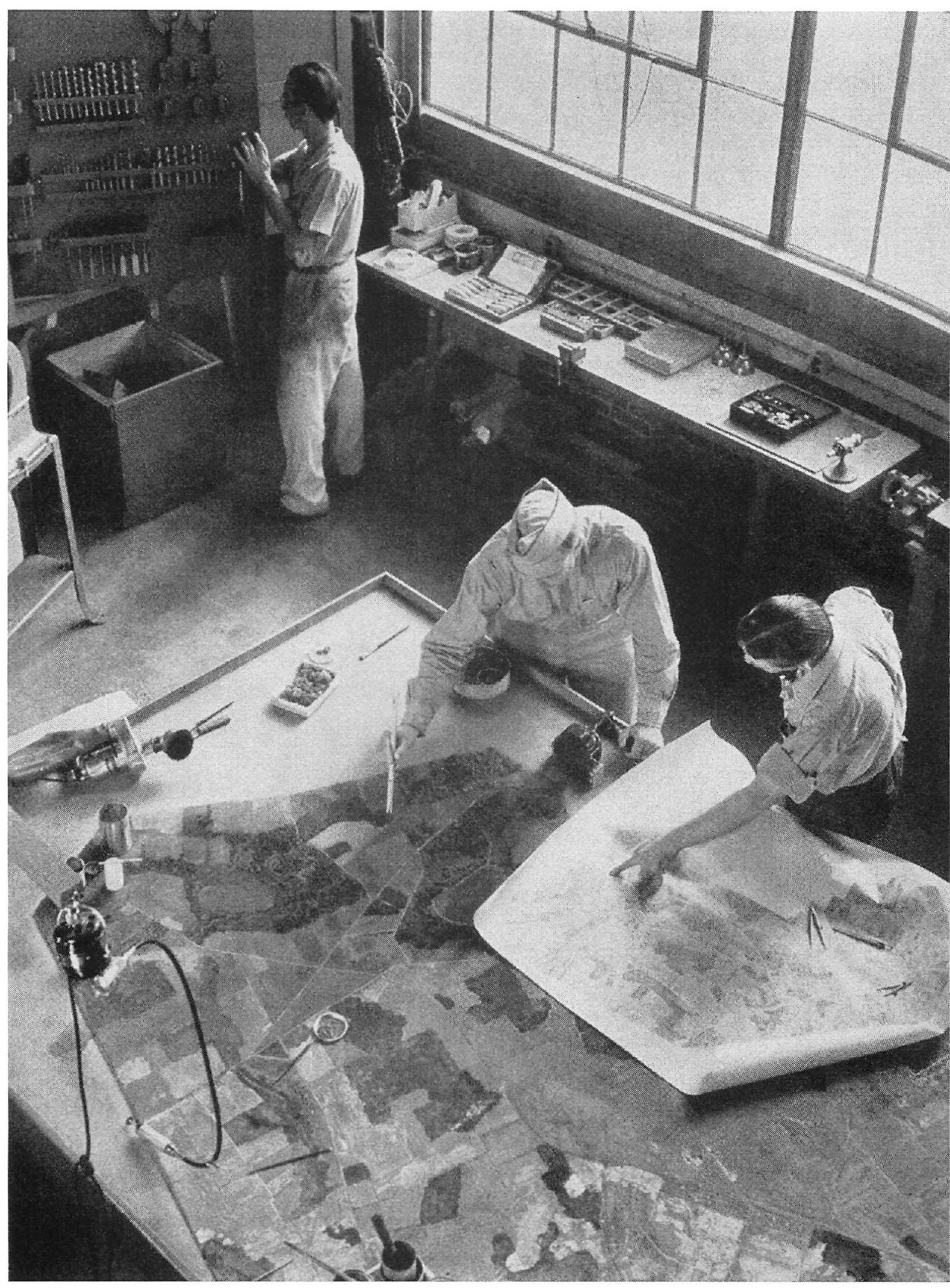




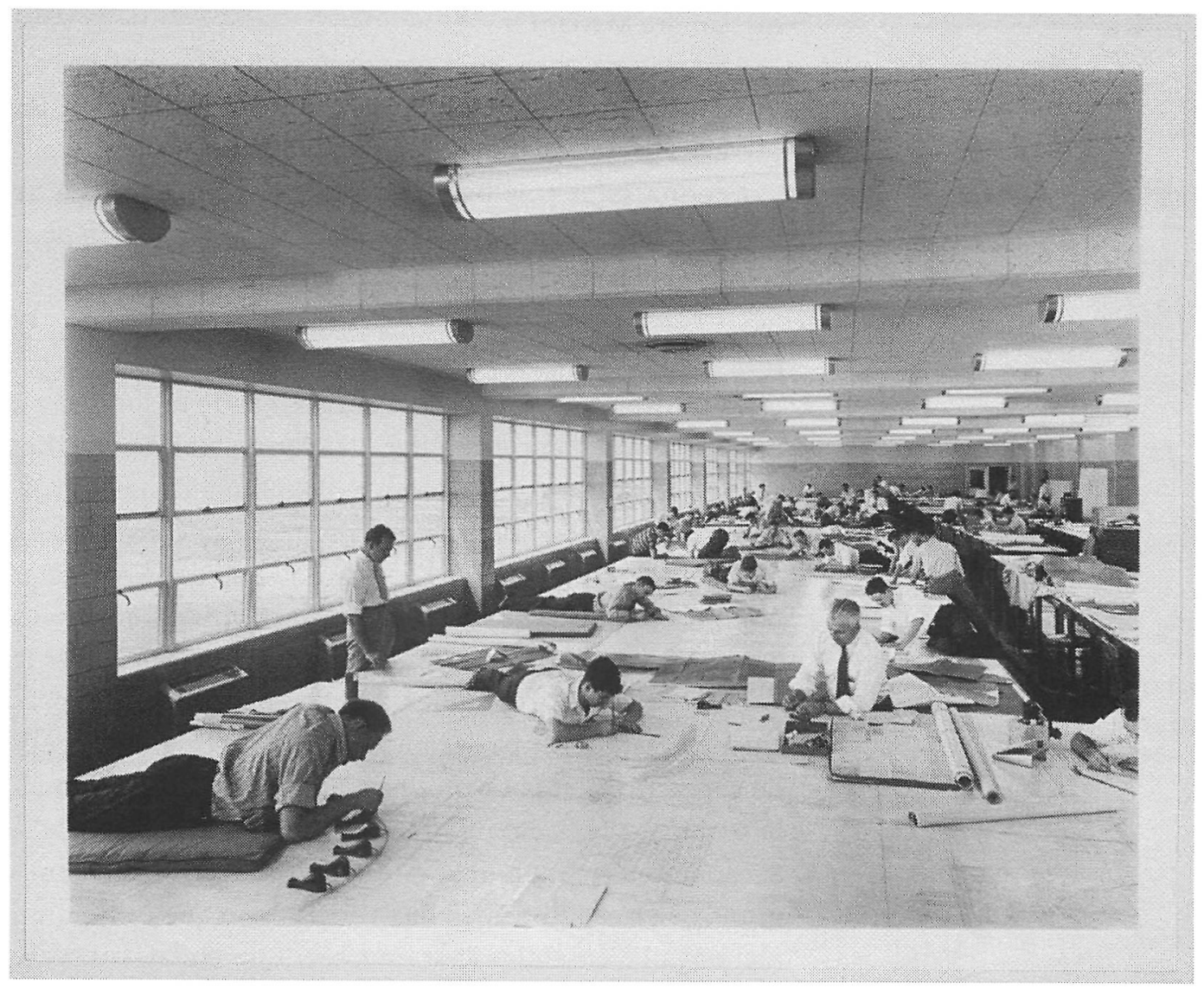

Usine de bombardiers de Ford Motor, Willow Run, Michigan, par Albert Kahn Associates, vue de l'atelier de dessin, 1942. Photographie de Hedrich-Blessing. Collection CCA. Don de Federico Bucci. Centre Canadien d'Architecture, Montréal. Don de Federico Bucci. o Chicago History Museum, HB-07074-C.

Une équipe de camoufleurs au travail à Fort Belvoir, Virginie, illustration dans Robert P. Breckenridge, Modern Camouflage: The New Science of Protective Concealment, 1942. McCill University Library, Montreal. 


\section{Alessandra Mariani}

Monsieur Cohen, vous êtes professeur d'histoire de l'architecture des $\mathrm{XIX}^{\mathrm{e}}$ et $\mathrm{XX}$ siècles, vous avez conçu des expositions et abondamment publié sur le sujet: vous connaissiez très bien cette lacune, cette brèche de I'histoire. Quelle a été l'ampleur de la recherche pour la combler?

\section{Jean-Louis Cohen}

Il y a environ une vingtaine d'années que je m'intéresse à ces questions, peut-être plus. J'ai commencé à travailler sur ces architectures (comme je l'explique dans l'avantpropos de la publication qui accompagne l'exposition) en étudiant l'architecture de l'occupation allemande et, pour être plus précis, celle réalisée en Alsace entre 1940 et 1944 et celle de l'occupation française en Allemagne après la guerre. J'ai ensuite travaillé sporadiquement sur différents thèmes, explorant par exemple l'architecture des camps de concentration pour un premier article, ou approfondi le problème de la domination américaine pour laquelle la Seconde Guerre mondiale est un épisode clé. Je crois toutefois que c'est au milieu des années 1990 que l'idée d'un réel travail d'ensemble s'est imposée. Tout en faisant beaucoup d'autres choses parce que je suis un être multitâche, au fil de recherches effectuées dans certains fonds d'archives en Allemagne, en Amérique ou en Russie, et au fil de mes enseignements, j'ai, touche après touche, constitué ce tableau.

II s'agit donc d'une recherche qui s'est étendue sur une vingtaine d'années?

J.-L. C. Oui, il aura fallu une vingtaine d'années, mais consacrées aussi à d'autres travaux, parce que bien souvent aller dans un fond d'archives pour une recherche amène à découvrir des choses qui servent pour autre chose. On ne trouve pas toujours ce que l'on cherche, on trouve ce que l'on ne cherche pas; c'est ce qui fait que la recherche est intéressante et que je m'en occupe moi-même. Je déteste déléguer la phase de l'exploration à des assistants : je préfère la faire moi-même, sauf quand j'ai vraiment repéré les documents et qu'il s'agit tout simplement de les reproduire. 
Je crois que c'est lorsqu'on découvre ce que l'on ne cherche pas qu'une joie intense se manifeste. Il arrive en quelque sorte que l'on soit découvert par les documents lorsqu'on est bien concentré sur un projet et que l'on devienne en quelque sorte radioactif au milieu d'une bibliothèque.

\section{Comment l'idée de l'exposition Architecture en uniforme s'est-elle manifestée?}

J.-L. C. Très tôt. Lorsque j'ai commencé à formuler ce dessein, je venais de réaliser l'exposition Scènes de la vie future'. C'était en 1995 et j'avais apprécié la productivité du Centre Canadien d'Architecture (CCA) et la passion qui l'animait. Ma première note sur cette future exposition fut envoyée à Nicholas Olsberg qui était à l'époque le conservateur en chef de l'institution. Ensuite le projet s'est un peu perdu et je l'ai fait figurer dans le programme que je développais pour la Cité de l'architecture et du patrimoine à Paris. Si je n'avais pas été écarté de cette institution en 2003, j'aurais certainement réalisé l'exposition à Paris.

\section{Comment s'intègre-t-elle aux programmes du Centre Canadien d'Architecture?}

J.-L. C. Je crois qu'il n'y a pas lieu de parler d'Architecture en uniforme en termes d'intégration à une programmation préétablie au CCA, parce que l'institution n'est pas structurée ainsi. Le programme général du CCA est surtout orienté sur l'exploration de questions, de problématiques nouvelles et sur une manière tout aussi originale de les aborder. L'exposition 1973: Désolé, plus d'essence ${ }^{2}$, par exemple, était en ce sens admirable parce qu'en se plaçant sur une conjoncture très particulière elle faisait spontanément émerger les origines du discours de l'architecture durable. Les expositions du CCA tendent à explorer les tâches blanches et à revenir sur les zones obscures pour mettre à jour des expériences historiques sans pour autant avoir l'illusion d'y trouver des enseignements toujours utiles; c'est de cette façon qu'elles éclairent les situations actuelles. La volonté d'explorer des épisodes non convenus, de privilégier les thèmes et la réflexion sur un sujet donné, plutôt que de présenter des figures héroïques d'architectes (il n'y a pas de monographies d'architectes au CCA) démontre bien,
Scènes de la vie future. L'architecture européenne et la tentation de l'Amérique 1893-1960 a été présentée du 14 juin au 24 septembre 1995 au Centre Canadien d'Architecture. L'exposition démontrait la fascination des architectes européens face aux progrès techniques, aux notions de productivité et aux nouveaux modèles d'urbanisme américains. 2

L'exposition 1973: Désolé, plus d'essence, présentée du 14 juin au 24 septembre 1995 au Centre Canadien d'Architecture, analysait l'innovation architecturale générée par la crise de l'essence de 1973. 
selon moi, le caractère fort de l'institution. Maintenant, si nous convenons qu'un programme peut être défini de cette façon, il est très clair qu'Architecture en uniforme s'y intègre parfaitement.

Une grande partie de nos lecteurs l'ignorent, mais de 1998 à 2003 le ministère de la Culture vous a mis en charge de l'élaboration et de la direction du projet de la Cité de l'architecture et du patrimoine au palais Chaillot à Paris. Pouvez-vous nous en dire un peu plus sur cette expérience?

J.-L. C. Après avoir déploré pendant des années l'absence d'une institution culturelle et d'une bibliothèque spécialisée sur l'architecture à Paris, j'ai été invité à passer à l'acte par le gouvernement socialiste de Lionel Jospin en 1998. J'ai donc monté un projet qui était en fait la recomposition de deux institutions existantes, la première étant le musée des Monuments français qui se trouvait dans le palais de Chaillot (et avant, dans le palais du Trocadéro) et qui était un musée de moulages essentiellement consacré à l'architecture médiévale et la seconde, l'Institut français d'architecture (une institution de petite taille dans un hôtel particulier de Saint-Germain-des-Prés), qu'il s'agissait d'articuler pour constituer un centre culturel consacré à l'architecture associant une réflexion sur le patrimoine, l'histoire et les pratiques contemporaines. J'ai développé ce projet et je crois que sans mon action de résistance au sein de multiples réseaux, la Cité n'aurait jamais vu le jour, ou à tout le moins pas dans la forme où nous la connaissons aujourd'hui. Le travail que j'ai mené a fait en sorte que le musée des moulages (antérieurement le musée des Monuments français) a finalement été rénové ; sans ténacité, il n'y aurait pas eu d'institution centrée sur l'architecture à Paris. Évidemment, par la suite, beaucoup d'idées que j'avais pour la création d'une nouvelle section dans le musée consacrée au XXe siècle et pour les programmes ont été abandonnées, amoindries ou tout simplement atténuées par mes successeurs, mûs pour certains par l'appétit du pouvoir et pas engagés dans les contenus que demandait un projet de cette envergure. Cet épisode reste donc pour moi un moment un peu mélancolique, mais l'institution 
est toujours là, elle se développe et est devenue un élément du paysage culturel parisien. De ce point de vue, il s'agit en effet d'un succès.

Êtes-vous d'accord sur la façon dont le projet s'est développé après votre départ?

J.-L. C. Je ne souhaite pas accabler ceux qui m'ont succédé, certaines personnes ont d'ailleurs travaillé avec moi et ce sont des personnes que je respecte: mais dans l'ensemble c'est malheureusement devenu un endroit très ennuyeux, où l'imagination ne règne pas, malgré le potentiel de l'endroit, qui demeure très grand.

Vous avez fait allusion - au cours de la conférence inaugurale du 13 avril dernier au CCA - au fait que chaque recherche pouvait être un travail psychanalytique inconscient. Vous préférez cependant dans le cas d'Architecture en uniforme parler de contre-transfert. Pouvez-vous préciser cette idée?

J.-L. C. Il s'agit d'une idée de la clinique freudienne. La notion de transfert est bien connue; elle se définit par le rapport qu'entretient un patient avec l'analyste, un rapport de parole, un rapport affectif qui permet la régression et la déconstruction par le patient de son vécu : c'est l'analyse vue par le patient. Du côté du thérapeute, il y a un rapport symétrique et réciproque, qui est le contre-transfert ou son rapport avec l'analysant. Ce contre-transfert lui permet d'opérer une mise à jour qui génère ses réponses, ses réactions et favorise le développement de la cure. Si cette métaphore du contre-transfert est appliquée à la recherche, elle rend compte du rapport qu'ont les chercheurs avec l'objet de leurs investigations, et celui-ci se révèle être toujours un rapport affectif. On ne passe pas sans raison quinze ans à travailler sur une question tout comme on ne garde pas un patient quinze ans dans son cabinet simplement parce qu'il paie ses séances.

Ce que je veux dire lorsque je parle de contre-transfert, c'est qu'il y a un attachement à ce thème lié à mes expériences d'enfant, au cadre historique dans lequel j'ai grandi, aux questions que je me pose depuis longtemps 
sur l'articulation entre la politique et l'architecture et ainsi de suite. Ce sont bien entendu des questions personnelles et non d'ordre scientifique qui m'ont entraîné vers cette recherche, comme cette volonté de combler une certaine lacune. La part de subjectivité est indispensable dans ce processus : c'est d'ailleurs une dimension qui me paraît fondamentale, par exemple dans le cadre des études doctorales. J'essaie en tant qu'enseignant, dans la mesure du possible, de toujours clarifier la relation des étudiants avec leur thème de recherche lorsqu'ils entreprennent ce cheminement qui consommera cinq années de leur vie et qui les définira en tant que jeunes intellectuels.

Comment avez-vous conceptualisé l'exposition, comment avez-vous construit son récit dans l'espace?

J.-L. C. J'ai construit mon propos en le racontant. Je suis quelqu'un qui passe beaucoup par l'expression orale. Au travers de conférences ou de séminaires, j'ai rassemblé thématiquement une première note d'intention en 1996. J'ai donc d'abord construit des cours et c'est à travers le récit des séances que j'ai pu délimiter une douzaine de thèmes qui ont fait leur chemin jusqu'aux galeries du CCA. J'ai ensuite rassemblé des images, des matériaux d'archives et identifié des protagonistes. Je me suis ainsi retrouvé par après avec ces douze thèmes et les matériaux surabondants qu'ils rassemblaient, qu'il s'est agit de faire entrer en quelque sorte avec un chausse-pied dans le vestibule et les six galeries du CCA.

Si une certaine densité de l'information transpire de l'exposition, c'est qu'il m'a été impossible de réduire les thèmes de douze à six pour "coller" au nombre des galeries. Le propos que j'y ai construit n'est par ailleurs pas diachronique : il aurait été absurde et vain de travailler uniquement sur des séquences temporelles. Il y aurait eu sans doute bien d'autres façons de la concevoir comme, par exemple, de former des thématiques nationales telles que Russie, Allemagne, Amérique, Angleterre, etc., mais, finalement, la voie de la thématique opératoire était celle qui permettait une mise en place plus efficace tout en révélant les liens les plus riches. Ces thématiques tissent entre elles un fil 
historique: la dernière salle est aussi celle de la fin de la guerre; c'est ainsi un horizon nouveau qui se profile au terme de l'exposition.

Il était important d'orchestrer l'espace de façon à ce que la démonstration soit intelligible, de représenter la scène sur laquelle se sont déroulés les événements et souligner le fait qu'il s'agissait d'une guerre essentiellement urbaine, industrielle et mobile. Il fallait ensuite présenter les protagonistes (je souhaitais depuis les tout débuts qu'il y ait une galerie de portraits); passer aux questions des confrontations, de la guerre aérienne vue du sol et de la protection, tout comme celles de la guerre aérienne vue d'en haut et de la production des techniques de camouflage. Il fallait aussi tenir compte de la transformation de l'échelle des projets, tout autant que de leur nature. Il fallait enfin imaginer une sortie où les avenirs imaginés pendant la guerre seraient confrontés avec ceux qui allaient être plus concrètement orchestrés par les plans.

Architecture en uniforme fait état de l'u effort de guerre", de la recherche, de la découverte et du déploiement des connaissances qui ont débouché vers une réorientation des politiques de l'économie mondiale. Parmi les nombreuses innovations recensées dans l'exposition, quelles sont selon vous celles dont l'impact a été le plus significatif?

J.-L. C. Il y en a plusieurs si l'on se pose du point de vue de l'architecture au sens strict du terme. La plus importante est à mon avis la "big box", l'usine fermée qui est un des grands types architecturaux $\mathrm{du} \mathrm{XX}^{\mathrm{e}}$ siècle. Il est clair que son origine est américaine: elle apparaît vers 1941-1942. Il s'agit d'un apport typologique, de l'éclosion d'un nouveau type d'édifice. On voit aussi l'apparition de la climatisation, de l'éclairage fluorescent et des matériaux légers de l'enveloppe. Un deuxième thème qui est tout aussi marquant est à la fois celui de l'utilisation de matériaux nouveaux et celui de l'utilisation nouvelle de matériaux anciens. Pensons à la transformation de l'usage du bois que l'on emploie désormais en petites sections autant dans la construction aéronautique que dans celle des bâtiments, 
parce que les colles phénoliques ont été mises au point à cette période. La colle n'est pas un matériau qu'on a l'habitude de célébrer (on loue volontiers l'acier, le béton, etc.); et pourtant il s'agit d'un matériau qui permet de créer de grands éléments à partir de petits segments de bois, tels que des nez de planeur, des charpentes, des panneaux de construction ou des avions de combat. Ces exemples, qui de prime abord ne semblent pas considérables, permettent en fin de compte de créer des monuments. Une troisième découverte est celle des systèmes préfabriqués comme le système MERO, qui aura un grand succès après la guerre. Ces systèmes conçus pour une guerre fondée sur la mobilité et le déplacement de milliers de personnes avec leur matériel à l'échelle de la planète ont vu leurs modes d'emploi recyclés à d'autres fins par la suite.

Si l'exposition présente en large partie l'importance de l'aspect fonctionnaliste de la production architecturale de cette période, la dernière salle offre un aspect polyphonique plus "globalisé" d'un éventuel après-guerre. Peut-on déjà y lire, selon vous, les prémices du postmodernisme architectural?

J.-L. C. Pour moi, le postmodernisme n'est pas un concept des années 1980 . On peut dire qu'il y a eu plusieurs postmodernismes au $\mathrm{XX}^{\mathrm{e}}$ siècle ou plusieurs démarches qui ont réagi contre ce qui était considéré comme la "pauvreté " ou les limites de l'architecture moderne.

Le régime soviétique des années $1930 \mathrm{a}$, par exemple, réagi contre l'architecture constructiviste pour des raisons politiques que je ne n'explorerai pas ici, mais il s'agit incontestablement d'un postmodernisme, puisqu'il y a alors refus des langages trop abstraits des Modernes débouchant vers une régression. Lorsqu'un des fondateurs du groupe de Stijl - l'architecte néerlandais Jacobus Johannes Pieter Oud conçoit le siège social de la Shell à La Haye en 1938, sans qu'il n'ait aucun rapport avec le régime stalinien, c'est un bâtiment postmoderne qu'il réalise. On peut donc avancer qu'il existe toute une série de cycles régressifs et que celui qui émerge en Russie et ensuite en Europe de l'Est à la fin de la guerre est un cycle fondé sur la célébration d'une 
tradition nationale lue d'une manière très particulière. $\mathrm{Ce}$ courant peut être mis en parallèle avec plusieurs reconstructions traditionnalistes qui sont aussi érigées en Europe de l'Ouest. Certaines des reconstructions de l'après-guerre en France sont très conservatrices et vont dans le même sens que plusieurs reconstructions allemandes qui visaient à reconstituer des petites villes médiévales. Sans affirmer que ces édifications sont postmodernes en tant que telles, il est clair qu'il y a une dimension régressive: on se console, on essaie de panser les plaies, on tente de trouver des éléments d'identité nationale ou régionale perdue; il s'agit en fin de compte de démarches rassurantes, réconfortantes.

Vous considérez ce genre de manifestation comme une régression?

J.-L. C. J'emploie le terme de régression ici dans un sens presque aussi freudien; il s'agit pour moi d'un retour à une phase antérieure. Je n'ai pas du tout l'intention de célébrer l'image progressiste de l'architecture, cette notion du progrès à travers les âges. Mais il est clair qu'il s'agit de retour en arrière dans l'utilisation de formes historiques. Vous avez par ailleurs dans cette architecture de l'après-guerre tout un volet de projets qui foncent dans l'autre direction, vers l'utilisation esthétique des nouvelles technologies. Il ne faut pas se cacher que les questions d'architecture et de l'esthétique sont présentes dans le volet culturel de la guerre froide. Elles s'annoncent déjà dans la reconstruction, bien que les choses soient très complexes. Ainsi, pendant un certain temps, les Russes pensaient recourir aux modèles américains lors de la reconstruction, avant que cette idée soit abandonnée.

Vous dépeignez dans l'exposition un architecte stratège, administrateur et politique. La période de la Seconde Guerre représenterait-elle un autre seuil pour la pratique?

J.-L. C. Dire que le goût change peut sembler simpliste, mais il est vrai que l'architecture moderne, qui était expérimentale avant la guerre, doit composer - lors de la fin de celle-ci - avec les enjeux des programmes massifiés de la reconstruction et de la construction des villes nouvelles. Il 
se passe des choses intéressantes dans la pratique à cette époque et plusieurs aspects sont à considérer. Une première considération serait celle de la taille des agences. Sans être une réalité nouvelle, le développement des grandes agences qui s'était arrêté avec la Crise de 1929 reprend et l'augmentation de leur taille transforme la pratique. Le développement d'agences d'envergure telles que Skidmore, Owings and Merrill (SOM) est lié aux programmes de guerre. Une autre considération est celle de la féminisation de la pratique, et il serait intéressant de consulter les statistiques afin d'en connaître exactement l'ampleur. Ce qui est certain en tout cas, c'est qu'aux États-Unis et peut-être au Canada aussi - mais je manque de données -, les femmes sont embauchées de manière assez sérieuse dans les agences d'architecture. Poursuivent-elles leur profession après la guerre ou abandonnent-elles, comme c'est le cas dans les usines, pour rendre le travail aux hommes qui reviennent du front? Voilà une autre question qu'il serait intéressant d'explorer. Une troisième considération, assez bien documentée dans la publication qui accompagne l'exposition, est l'idée de la pratique expérimentale: j'y fais allusion lorsque je cite l'excellent article du scientifique anglais John Desmond Bernal. Les architectes qui ont travaillé sur le camouflage ou pour l'armement reviennent de la guerre moins "artistes"; ils sont plus gestionnaires, chercheurs et sociologues. L'architecte britannique Hugh Casson révèle que des phénomènes inattendus sont générés par les travaux sur le camouflage réalisés au cours de la guerre: il relève le retour de l'emploi de la couleur dans la conception architecturale, s'opposant à l'architecture moderne de l'avant-guerre, essentiellement blanche. Enfin, un autre aspect significatif pour la pratique professionnelle est celui des réseaux créés au cours de la guerre qui génèrent une fraternité qui influencera fortement le système de la commande par la suite, les vieux camarades de front ne manquant pas de faire appel les uns aux autres.

Quel écho croyez-vous qu'une telle exposition peut avoir dans un pays qui n'a pas été un témoin direct des événements? 
J.-L. C. Même si le Canada n'a pas été directement atteint sur son territoire, il a quand même été transformé. Je ne suis pas spécialiste de l'histoire du Canada, et le temps a manqué pour approfondir la question, mais je crois que le Canada en tant que nation a été soudé par sa participation aux deux grandes guerres du $\mathrm{XX}^{\mathrm{e}}$ siècle. Par ailleurs, je pense aussi que le public qui sera sensible à l'exposition comme à la publication comprendra que beaucoup d'éléments de la transformation du paysage architectural et de la pratique ont été déterminés par les expériences américaines et par la circulation des images. Il me semble que ces composantes ont eu un impact certain sur la scène canadienne.

Le CCA annonce que l'exposition circulera dans deux autres pays: peut-on envisager que l'effet sera différent ailleurs?

J.-L. C. Dans l'hypothèse d'une présentation à Rotterdam par exemple, qui est la première ville après Varsovie en 1939 à être rasée par un bombardement, l'impact devrait être assez significatif. Elle devrait être aussi présentée à Rome, qui a subi la guerre et le fascisme: dans un cas comme dans l'autre, de nouvelles références (tant au niveau des documents que des extraits filmiques) serviront à donner une couleur locale au récit. Dans un tel processus de " réexposition", il faudra aussi, bien entendu, produire dans ces lieux un discours alternatif qui assure la distance critique nécessaire.

Au-delà des limites pédagogiques et didactiques, Architecture en uniforme propose aussi une lecture qui renvoie à l'aspect éthique de la pratique architecturale. Les expositions d'architecture ont très souvent endossé un rôle prescriptif: le résultat est-il, dans ce cas, fortuit ou intentionnel?

J.-L. C. Poser la question c'est y répondre... Je ne crois pas que l'on puisse considérer qu'il soit totalement fortuit ; en même temps, je me suis efforcé de ne pas construire une exposition lourdement didactique. J'ai cherché à la rendre parfois allusive afin de laisser les gens réfléchir et décider pour eux-mêmes. L'enjeu de cette exposition n'est pas de 
préconiser une esthétique: elle est porteuse d'un message éthique et moral qui n'est pas pilonné à coups de pièces d'artillerie. Elle rappelle que l'architecture est un exercice éminemment lié au politique et à la politique et qu'aucun professionnel aussi formaliste soit-il ne peut se dérober à cette dimension. Elle soulève le fait que la pratique de l'architecture dans les sociétés actuelles pose toujours des questions morales et éthiques. Pour qui travaille-t-on? Travaille-t-on pour une dictature, pour la propagande ou pour des destinataires qui méritent que l'on s'occupe d'eux?

\section{Enfin, quels liens cette exposition peut-elle établir avec notre contexte actuel?}

J.-L. C. On se pose de telles questions aujourd'hui par exemple lorsqu'on travaille en Chine. Quel sens y a-t-il à travailler pour un capitalisme d'État totalitaire? Mais on sait aussi que les pyramides n'ont pas été construites par un régime démocratique et qu'elles constituent cependant un jalon dans notre culture... Rien n'est simple, de toute évidence: l'exposition devrait néanmoins permettre de réfléchir sur ce que cela implique de réaliser des projets dans des sociétés en conflit, que ceux-ci soient externes ou internes; des sociétés qui étaient, sont et resteront toujours des sociétés de classe.

Aujourd'hui les choses sont très différentes. Fidèle à la leçon de mon maître et ami Manfredo Tafuri, je me garderai bien de faire de l'histoire opérative, c'est-à-dire de penser qu'il y a des leçons directes à tirer de tout cela. Les sens générés sont habituellement indirects et il faut relativiser. Pourquoi est-il possible de raconter ces histoires autour de la Seconde Guerre mondiale? Parce que c'était une guerre où toute la société était impliquée. Les guerres aujourd'hui mobilisent des mercenaires, des spécialistes et des techniciens; il s'agit donc d'événements fort différents. Il est vrai qu'elles continuent à mobiliser des expertises spatiales, organisationnelles, constructives, visuelles et scientifiques, mais sans aller solliciter l'ensemble du tissu social comme cela a été le cas entre 1939 et 1945. De ce point de vue, cette guerre mondiale conserve une spécificité que tous les épisodes qui ont suivi n'ont pas eue. 


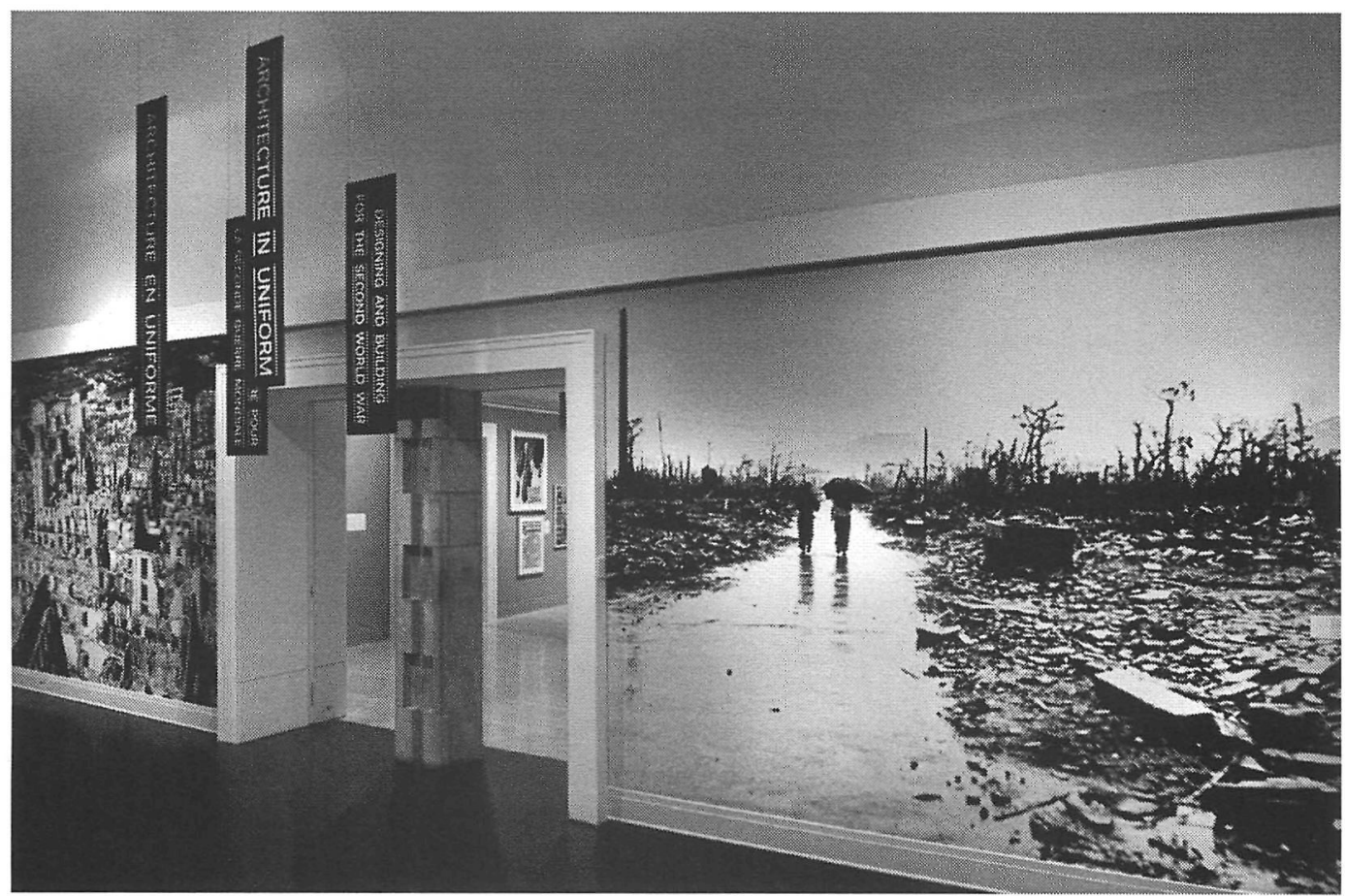

(Image ci-haut et suivantes) Vue de l'exposition du CCA, Architecture en uniforme: projeter et construire pour la Seconde Guerre mondiale. Centre Canadien d'Architecture, Montréal. 


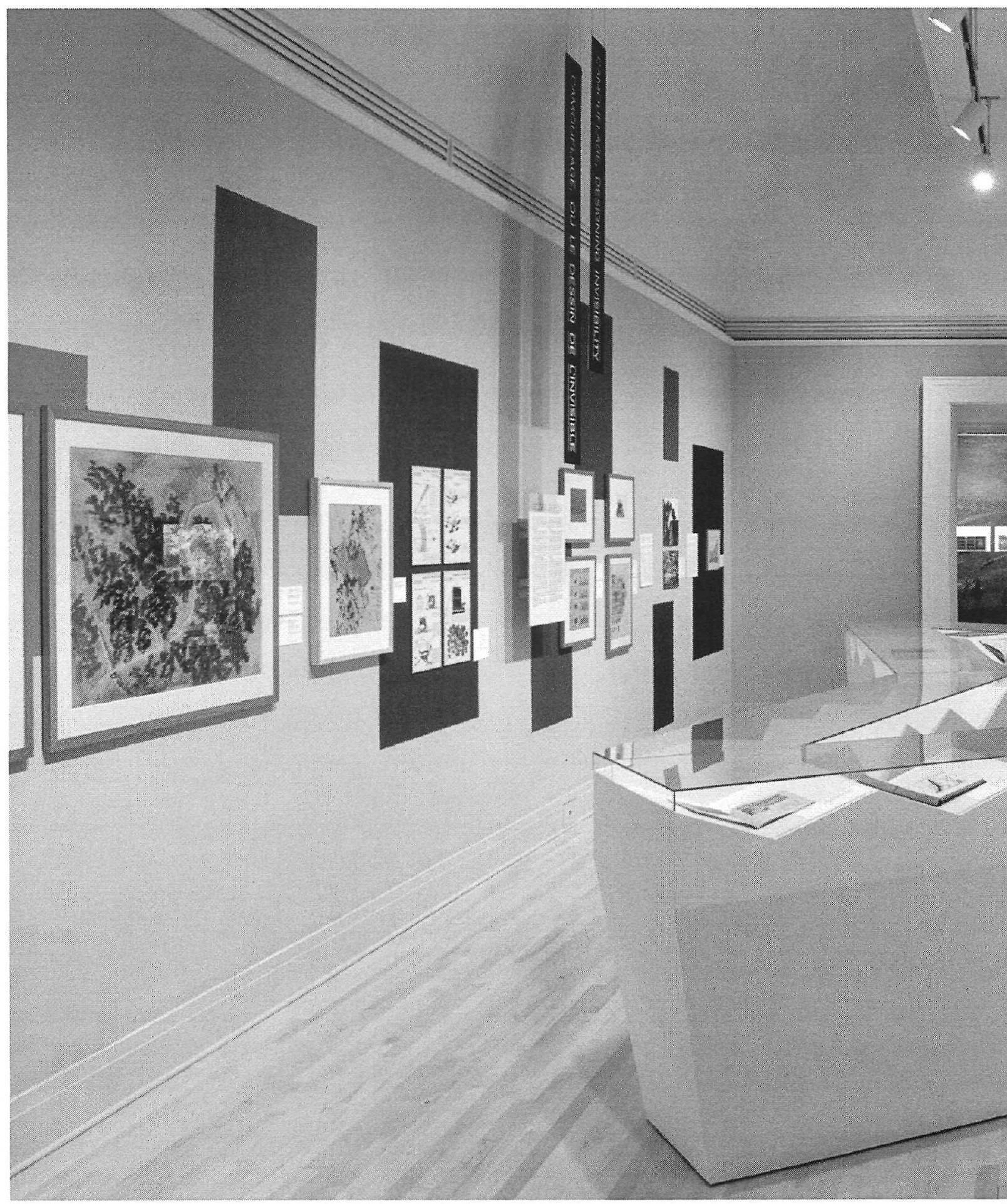




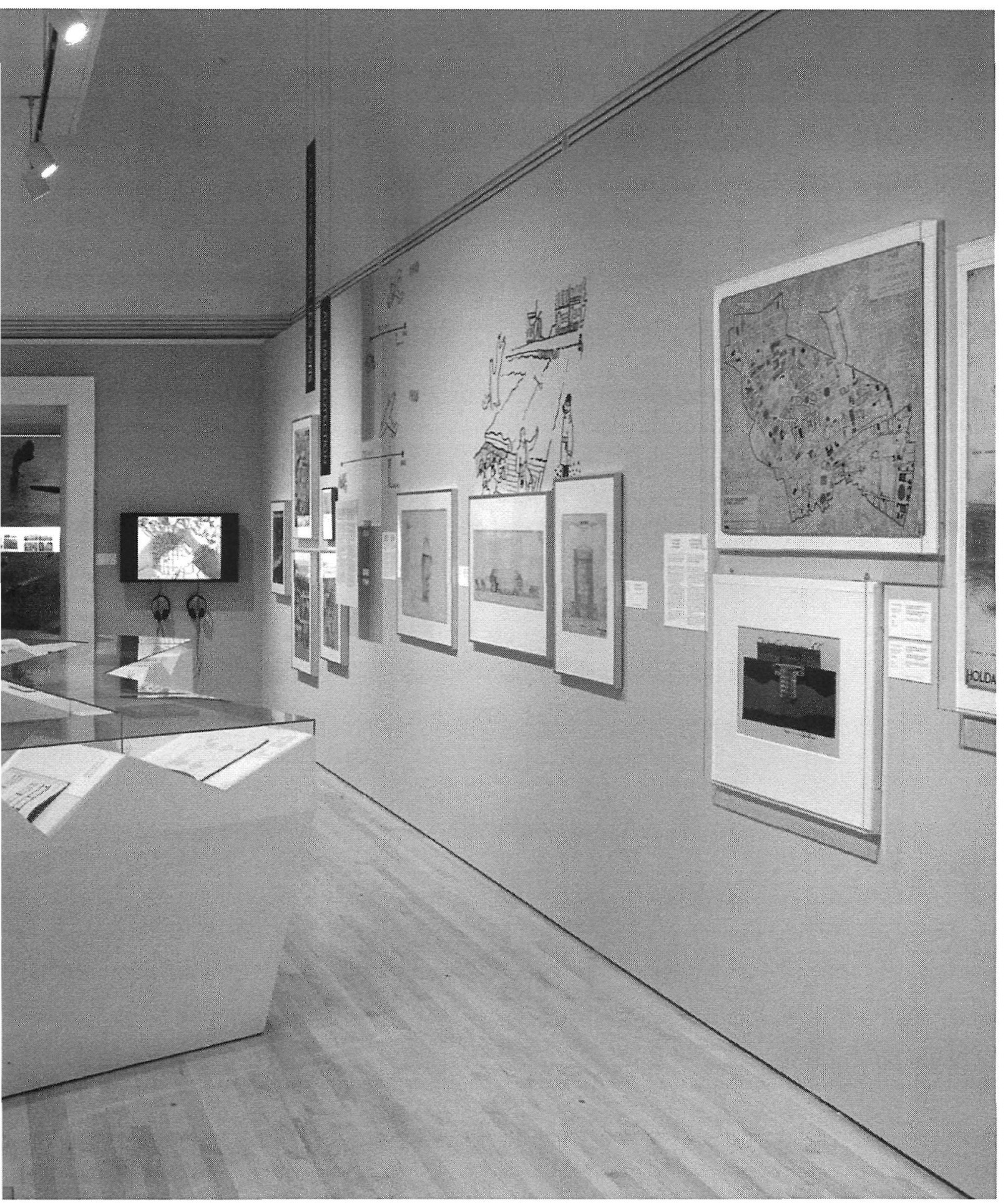




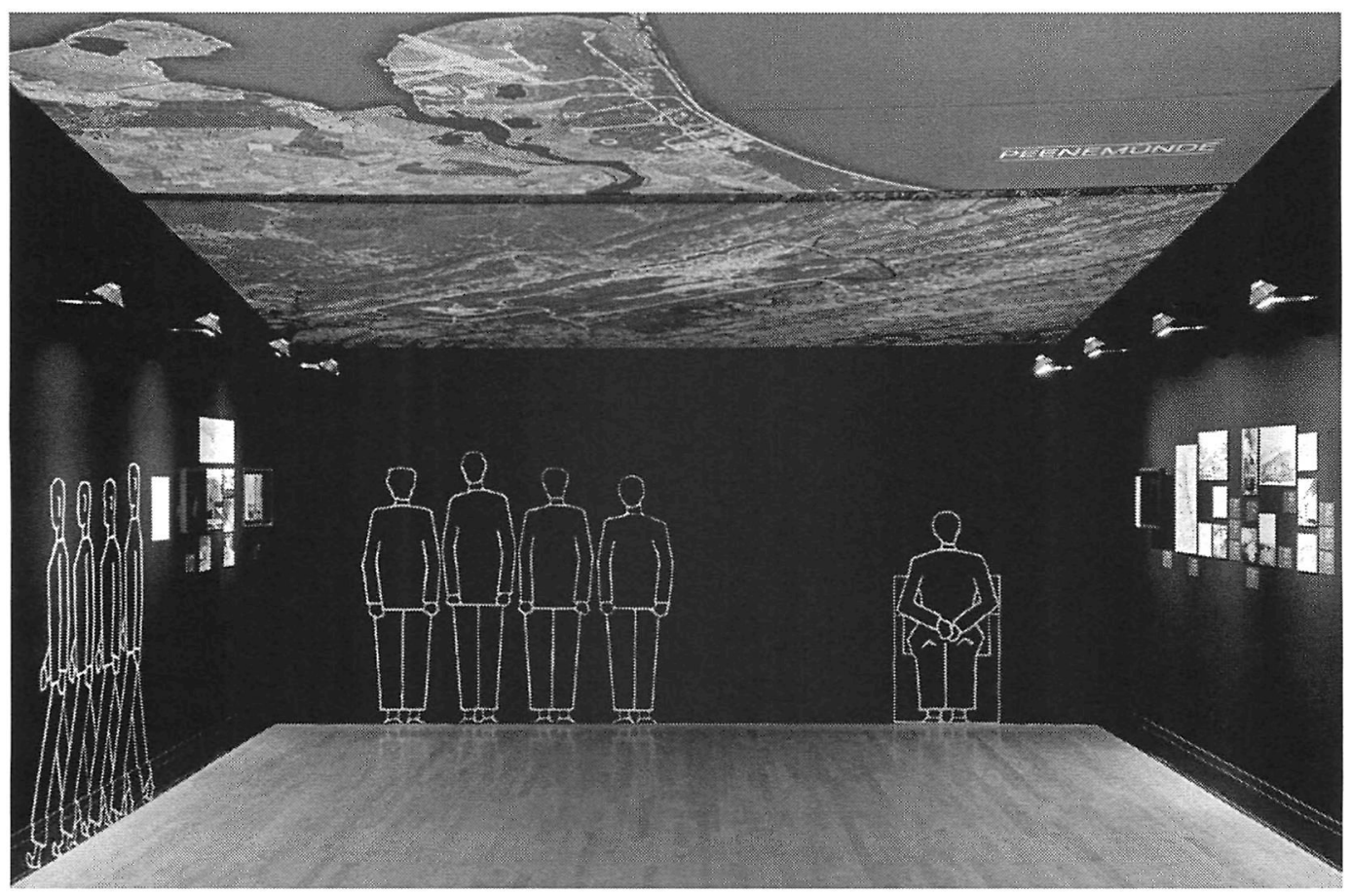




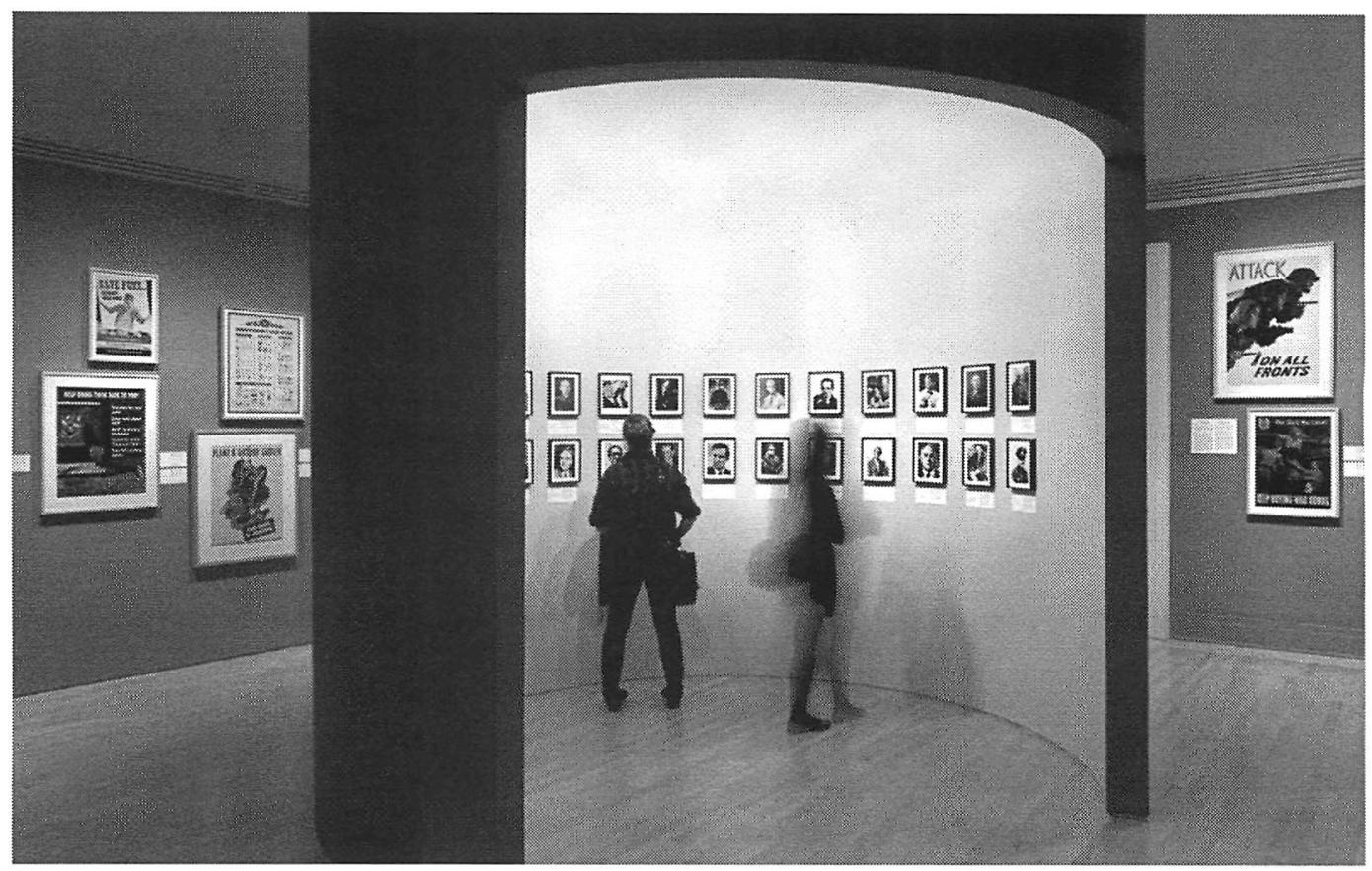

